\author{
K. Rebbas, E. Vela, A. F. Bougaham, A. Belharrat, G. De Belair \& R. Prelli
}

\title{
Découverte de Christella dentata (Thelypteridaceae) en Algérie
}

\begin{abstract}
Rebbas, K., Vela, E., Bougaham, A. F., Belharrat, A., De Belair, G. \& Prelli, R.: Découverte de Christella dentata (Thelypteridaceae) en Algérie. - F1. Medit. 29: 55-66. 2019. - ISSN: 1120-4052 printed, 2240-4538 online.

Christella dentata (= Cylosorus dentatus, Thelypteridaceae, Pteridophyta), native to the tropical and subtropical regions of Africa and Asia, has been observed between Jijel and Bejaia in northeastern Algeria. Distribution, ecological notes and comparison with related taxa as Cyclosorus interruptus (Dryopteris gongylodes p.p.) are also provided. This is the first report of this species in North Africa.
\end{abstract}

Key words: Pteridophytes, biodiversity survey, flora of Algeria, North Africa, Important Plant Area.

\section{Introduction}

Le littoral montagneux du tell littoral algéro-tunisien constitue un point chaud de biodiversité dénommé « Kabylies-Numidie-Kroumirie » (Vela \& Benhouhou 2007) et abrite de nombreuses zones importantes pour les plantes (Radford \& al. 2011; Yahi \& al. 2012). Cependant elles n'ont plus fait l'objet d'explorations botaniques depuis le milieu du $20^{\mathrm{e}}$ siècle, jusqu'à une période plus récente marquée par un renouveau des découvertes taxonomiques ou chorologiques d'importance nationale voire nord-africaine (cf. Rebbas \& Vela 2008; Martin 2008; Daoud-Bouattour \& al. 2009; Laribi \& al. 2009; Laribi \& al. 2011; De Belair \& Vela 2011; Haou \& al. 2011; Vela \& al. 2012a-2012b; Ouarmim \& al. 2013; Hadji \& Rebbas 2014; El Mokni \& al. 2015a, 2015b; Vela \& al. 2015) sans parler des nombreux xénophytes d'apparition et/ou de naturalisation récente (à titre d'exemple cf. Vela \& al. 2013).

Malgré une reprise des recherches botaniques sur le terrain, en Kroumirie tunisienne, en Numidie littorale algérienne, et en quelques points de Kabylie (Béjaïa, Akfadou) de nombreux secteurs demeurent sous-étudiés dont la Kabylie des Babors, région montagneuse difficile d'accès (voir néanmoins Gharzouli \& Djellouli 2005). Malgré une reprise des recherches botaniques sur le terrain, en Kroumirie tunisienne, en Numidie littorale algérienne, et en quelques points de Kabylie (Béjaïa, Akfadou) de nombreux secteurs demeurent sous-étudiés dont la Kabylie des Babors, région montagneuse difficile d'accès (voir néanmoins Gharzouli \& Djellouli 2005). 
A l'occasion des recherches sur la végétation rupicole, sources et suintement des rochers du littoral de petite Kabylie effectués dans le cadre des travaux d'une thèse de doctorat (cfr. Rebbas 2014), nous avons visité plusieurs reprises l'oued Ziama, situé à la limite administrative entre la wilaya de Béjaïa et celle de Jijel. Une première station a été découverte par l'un de nous (K.R.) en juillet 2007 sur un petit talus rocheux de la berge de l'oued au niveau d'une petite source. L'observation de jeunes pieds immatures de Pteris cretica et d'une fougère indéterminée nous encouragea à renouveler les prospections. Une nouvelle visite en août 2009, nous a permis de retrouver la première station, malheureusement les individus de la fougère inconnue, bien que mieux développés étaient toujours immatures, et l'individu de Pteris avait disparu suite à un petit éboulement. Quelques jours plus tard, en prospectant plus en amont, que nous avons découvert plusieurs individus bien développées directement dans la ripisylve de l'oued, et avons ainsi pu démarrer le travail d'identification. Ce n'est que plus récemment, pendant la préparation de cette publication, que deux autres stations, plus éloignées mais bien plus abondantes et mieux conservées, ont pu être découvertes en septembre 2018 et en mars 2019 (A.F.B. et A.B.), confirmant l'indigénat et la pérennité de l'espèce dans ce secteur.

Les flores classiques en usage en Algérie pour les Ptéridophytes (Maire 1952; Quézel \& Santa 1962) nous conduisaient systématiquement vers Dryopteris gongylodes subsp. propinqua (R. Br.) Christensen, à défaut d'autre candidat potentiel. En revanche, les flores d'Europe et notamment l'ouvrage spécialisé le plus usité par les botanistes francophones (Prelli 2001) nous conduisaient vers Christella dentata (Forssk.) Brownsey \& Jermy, là aussi à défaut d'autre candidat. D'après la synthèse récente de Dobignard \& Chatelain (2010) pour le nord de l'Afrique (incluant Madère et Canaries), le premier nom est désormais retenu sous le nom synonyme de Cyclosorus interruptus (Willd.) H. Itô, tandis que le second est un nom retenu mais a aussi pour synonyme le nom désuet Cyclosorus dentatus (Forssk.) Ching, ce qui montre bien, sinon leur parenté réelle, du moins leur parenté longtemps supposée et la délicatesse de leur distinction. Le plus remarquable dans cet état de fait était que les flores du Maghreb ignoraient le second (seul le premier étant présent), tandis que les flores d'Europe (Madère et canaries incluses) ignoraient le premier (seul le second étant présent). Notre découverte du second en Algérie avait aussitôt été signalée à Cyrille Chatelain et Alain Dobignard qui ont ainsi pu l'ajouter dans leur « Suppléments et corrigenda aux volumes 1 à $4 »$ (in Dobignard \& Chatelain 2013 : 380).

C'est suite à cela que nous détaillons ici l'identification de notre nouvelle fougère et que nous révisons la situation taxonomique et chorologique du genre Cyclosorus sensu lato (Thelypteridaceae) en Algérie, et par extension en Afrique du Nord puis en Méditerranée occidentale.

\section{Description des sites et du matériel étudié}

Les coordonnées sont fournies par GPS de localisation au sol. Les altitudes approximatives données par le GPS sont vérifiées et corrigées si besoin d'après un logiciel de cartographie avec modèle numérique de terrain. Les stations de Christella dentata sont les suivantes (Fig. 1) :

Algérie, Jijel, Ziama, Oued Ziama, $36.6533^{\circ} \mathrm{N}, 05.4541^{\circ} \mathrm{E}, 30 \mathrm{~m}, 20.08 .2009$, K. Rebbas; Algérie, Jijel, Ziama, Oued Ziama, $36.6536^{\circ} \mathrm{N}, 05.4530^{\circ} \mathrm{E}, 30 \mathrm{~m}, 20.08 .2009$, K. Rebbas; 


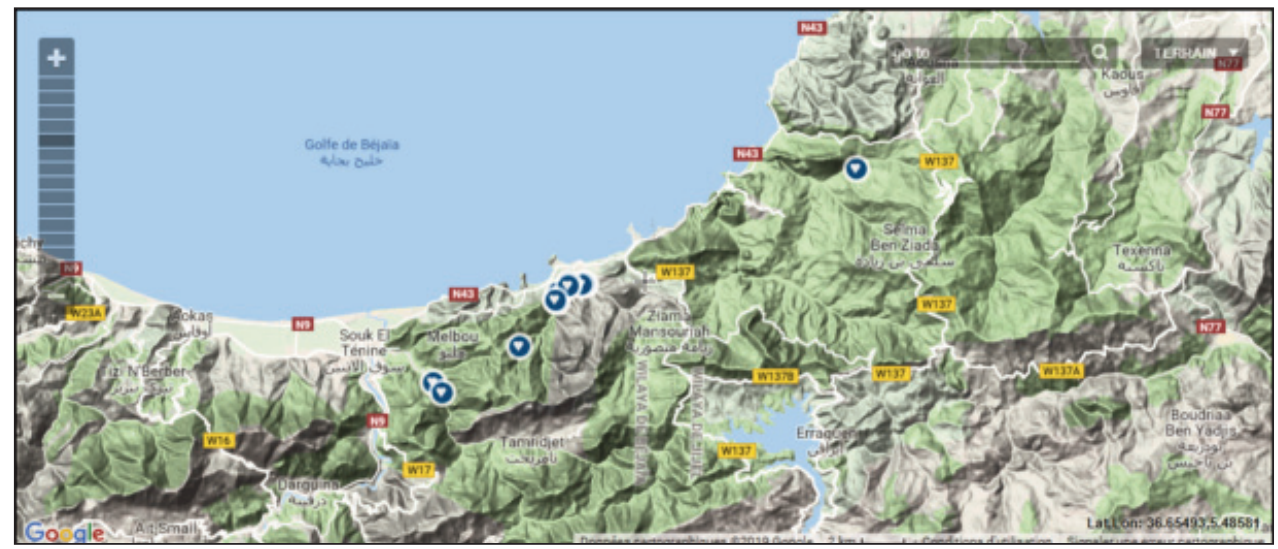

Fig. 1. Localisation géographique des stations de Christella dentata à l'est de la wilaya de Béjaia et à l'ouest de la wilaya de Jijel (carte réalisée avec GeoCAT, http://geocat.kew.org).

Algérie, Jijel, Ziama, Oued Ziama, $36.6536^{\circ} \mathrm{N}, 05.4511^{\circ} \mathrm{E}, 30 \mathrm{~m}, 20.08 .2009$, K. Rebbas; Algérie, Jijel, Ziama, Oued Akherkhor, $36.6531^{\circ} \mathrm{N}, 05.4448^{\circ} \mathrm{E}, 20 \mathrm{~m}, 18.12 .2018$, K. Rebbas;

Algérie, Jijel, Ziama, Oued Akherkhor, $36.6541^{\circ} \mathrm{N}, 05.4453^{\circ} \mathrm{E}, 20 \mathrm{~m}, 18.12 .2018, \mathrm{~K}$. Rebbas;

Algérie, Jijel, Ziama, Oued Akherkhor, $36.6504^{\circ} \mathrm{N}, 05.4397^{\circ} \mathrm{E}, 40 \mathrm{~m}, 23.12 .2018, \mathrm{~K}$. Rebbas;

Algérie, Jijel, Ziama, Oued Akherkhor, $36.6468^{\circ} \mathrm{N}, 05.4379^{\circ} \mathrm{E}, 50 \mathrm{~m}, 23.12 .2018, \mathrm{~K}$. Rebbas;

Algérie, Jijel, Ziama, Oued Akherkhor, $36.6462^{\circ} \mathrm{N}, 05.4392^{\circ} \mathrm{E}, 60 \mathrm{~m}, 23.12 .2018, \mathrm{~K}$. Rebbas;

Algérie, Jijel, Parc national de Taza, Oued Taza, T'bola, $36.70417^{\circ} \mathrm{N}, 5.60015^{\circ} \mathrm{E}, 170 \mathrm{~m}$, 11.09.2018, A.F. Bougaham et A. Bouchareb;

Algérie, Bejaia, Souk El Tenine, Oued Boulazazene, Taramanet, $36.6119^{\circ} \mathrm{N}, 5.3727^{\circ} \mathrm{E}$, 50m, 19.03.2019, A.F. Bougaham et A. Belharrat;

Algérie, Bejaia, Souk El Tenine, Oued Boulazazene, Gar Dourar, 36.6069 ${ }^{\circ} \mathrm{N}, 5.3777^{\circ} \mathrm{E}$, 60m, 19.03.2019, A.F. Bougaham et A. Belharrat.

L'oued Ziama en petite Kabylie représente un bassin versant incluant la face nord de l'Adrar El-Alem (1167 m) et du Djebel El-Kouf (1369 m), l'ensemble du Kef Es-Safsaf et la face ouest du Djebel Mrada $(617 \mathrm{~m})$. Son embouchure rejoint la Méditerranée entre les villes de Ziama Mansouriah et de Melbou (ex-Les Falaises). Les stations sont situées entre un et deux kilomètres de l'embouchure, sur l'oued Ziama et son affluent rive gauche l'oued Akherkhor, un peu avant la confluence de ces deux derniers. Il s'agit d'un fleuve côtier à débit permanent constitué d'eaux claires à courant modérée. Le lit est principalement recouvert de galets et par endroit de limons fins. Les berges sont couvertes par une ripisylve d'Aulne glutineux (Alnus glutinosa (L.) Gaertn.) formant une galerie dense et en très 
bon état de conservation. Nous avons dénombré environ deux centaines de touffes de Christella dentata, dont 110 sur la seule station la plus en amont.

La localité de T'bola se trouve le long de l'oued Taza dans la forêt domaniale de Guerrouche située au sein de la zone centrale du Parc national de Taza. La station est couverte par une forêt de Chêne zéen (Quercus canariensis Willd.) en bon état de conservation. Le sous-bois est assez diversifié avec principalement Viburnum tinus L., Laurus nobilis L., Alnus glutinosa (L.) Gaertn., Rubus incanescens (DC.) Bertol., Hedera algeriensis Hibberd, Asplenium onopteris L. Nous avons dénombré 130 touffes de Christella dentata sur une surface de $400 \mathrm{~m}^{2}$ (Fig. 2).

La troisième station de Christella dentata a été découverte dans une ripisylve s'étendant le long de l'oued Boulezazene (affluent de l'oued Agarioune) qui prend source de Djebel Tababort (1969 m.), située dans le village de Tizi War dans la commune de Melbou de la Daira de Souk El Tenine. Les deux localités à Christella dentata sont caractérisées par une formation végétale dominée par Alnus glutinosa (L.) Gaertn., Populus alba L. et Salix alba L. Cette espèce pousse le long des anciens canaux d'irrigation destinés à arroser les vergers abandonnés. Le sous-bois est formé principalement par Nerium oleander L., Rubus ulmifolius Schott., Dittrichia viscosa (L.) Greuter, Phragmites communis Trin, Hedera algeriensis Hibberd et Vinca major L. Nous avons dénombré 80 touffes de Christella dentata dans la localité Taramanet et 28 touffes dans les gorges Gar-Dourar.

Dans ces sites le climat est pluvieux avec des précipitations annuelles moyennes comprises entre 1000 et $1200 \mathrm{~mm}$ (A.N.R.H. 1993) mais méditerranéen car l'été reste sec, avec moins de $40 \mathrm{~mm}$ en moyenne cumulés sur les trois mois (juin, juillet et août). Il se situe dans l'étage bioclimatique humide à hiver chaud au sens de Daget (1977).

Le long de l'oued Ziama, cinq espèces de ptéridophytes sont présentes en mélange sur au moins huit stations distantes chacune de 100 à $200 \mathrm{~m}$. Il s'agit de: Adiantum capillusveneris L., Christella dentata (Forssk.) Brownsey \& Jermy, Polystichum setiferum (Forssk.) T. Moore ex Woyn., Pteris cretica L., P. longifolia L. (= P. vittata L.). La première (Adiantum) et la troisième (Polystichum) sont assez communes en Algérie, tandis que la dernière (Pteris longifolia) est signalée comme rare et présente, entre autres, en Petite Kabylie (Quézel \& Santa 1962) où nous la connaissons de plusieurs sites le long de l'oued Agrioun (cascade de Kefrida, sources de Chaabet El Akra...). Récemment, une nouvelle station de cette ptéridophyte a été découverte à Ath Yanni dans la région du Djurdjura septentrional et une autre dans la région de Toudja (Béjaia) par un des auteurs (K.R.). Pteris cretica est en revanche très rare en Algérie et présente pour toute l'Afrique du nord seulement en petite Kabylie, dans les montagnes de la wilaya de Jijel (Battandier \& Trabut 1905; Maire 1952; Quezel \& Santa 1962). Enfin si Cyclosorus interruptus (= Dryopteris gongyloides subsp. propinqua auct.; Nephrodium unitum subsp. callense Trab.) est très rare dans tout le Maghreb (connu jusqu'à présent en Algérie seulement en Numidie littorale au bord des lacs de la région d'El Kala, (Desfontaines 1799; Battandier \& Trabut 1905; Maire 1952; Quézel \& Santa 1962) et au Maroc seulement dans les marais du littoral atlantique près de Larache (Jahandiez \& Maire 1931; Maire 1952), Christella dentata (= Cyclosorus dentatus), quant à elle n'avait jamais été signalée au Maghreb, mais seulement des îles macaronésiennes (Azores, Madère, Canaries, Cap Vert), d'Espagne continentale (Andalousie, Galice), d'Italie (Campanie, Sicile) et de Crète (cf. Prelli 2001). 


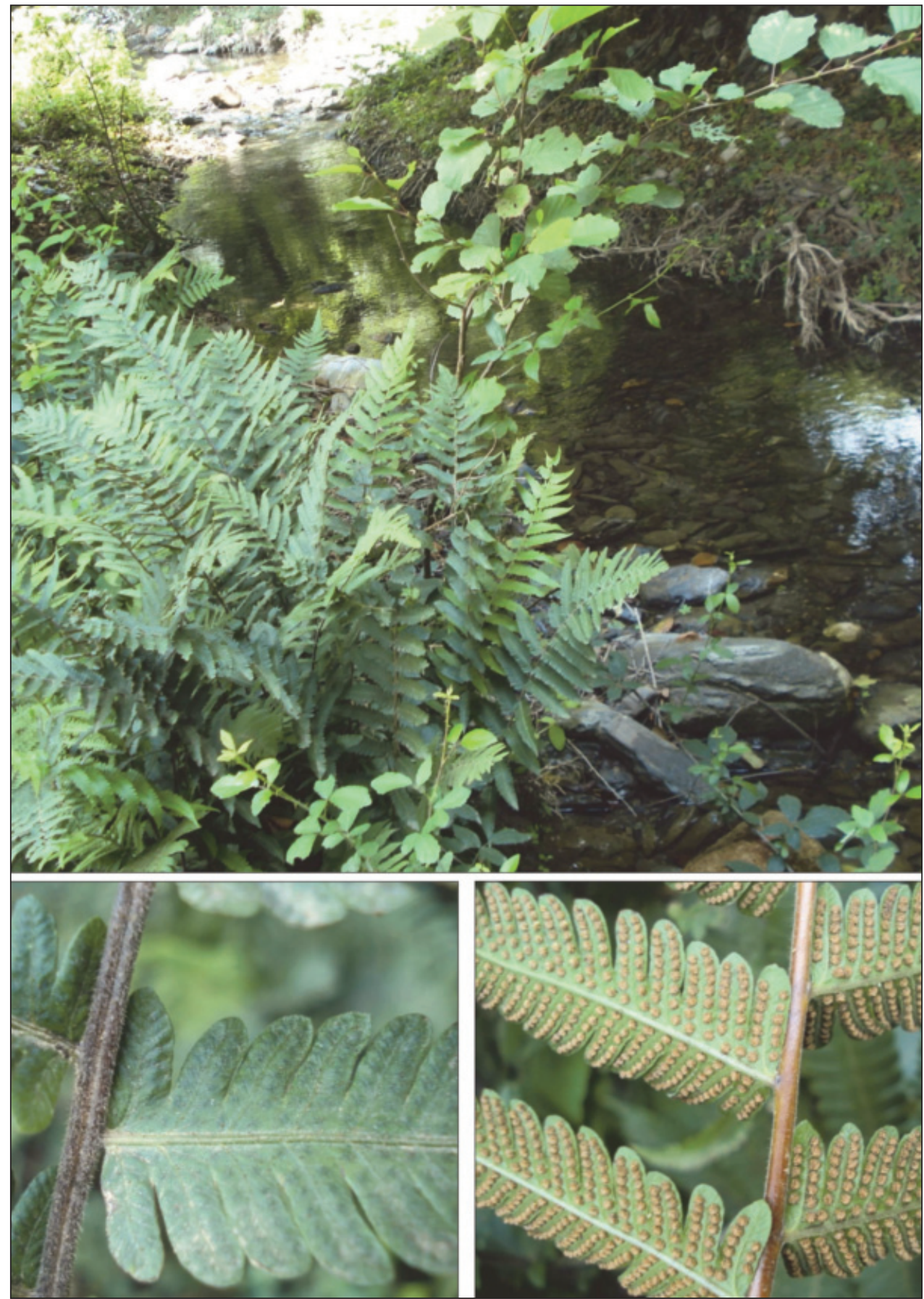

Fig. 2. Christella dentata. En haut : la plante et son biotope ; en bas : face supérieure et inférieure des pinnules (Photos: K. Rebbas, Oued Ziama, 20.08.2009). 
Le matériel récolté est déposé à MPU (Montpellier, France) et ENSA (El Harrach, Alger), et dans ce dernier pour majeure partie sous l'acronyme GDB intégré à ENSA. Deux copies (iso-récoltes) sont déposées à PAL (Herbarium Mediterraneum, Palerme, Italie). De plus, des photos (ph) complétant le matériel GDB sont également consultables à l'adresse http://gdebelair.com/tax/zzpz.html\#Thelypteridaceae [consulté le 23/12/2018]: Polystichum setiferum (Forssk.) Woyn., Algérie, Jijel, Oued Ziama x O. Akherkhour, 200908-20, K. Rebbas, hEV n0025 (MPU);

Christella dentata (Forssk.) Brownsey \& Jermy, Algérie, Jijel, Oued Ziama, 2009-08-20, K. Rebbas, hEV n0026 (MPU);

Christella dentata (Forssk.) Brownsey \& Jermy, Algérie, Jijel, Oued Ziama, 2009-08-20, K. Rebbas, hEV n0026bis (PAL);

Pteris cretica L., Algérie, Jijel, Oued Ziama x O. Akherkhour, 2009-08-20, K. Rebbas, hEV n0027 (MPU);

Pteris vittata L., Algérie, Jijel, Oued Ziama x O. Akherkhour, 2009-08-20, K. Rebbas, hEV $\mathrm{n}^{\circ} 0028$ (MPU);

Christella dentata (Forssk.) Brownsey \& Jermy, Algérie, Jijel, Oued Ziama x O. Akherkhour, 2009-08-20, K. Rebbas, hEV n0029 (MPU);

Christella dentata (Forssk.) Brownsey \& Jermy, Algérie, Jijel, Oued Ziama x O. Akherkhour, 2009-08-20, K. Rebbas, hEV n0029bis (PAL);

Christella dentata (Forssk.) Brownsey \& Jermy, Algérie, Jijel, Oued Ziama, $36.6541^{\circ} \mathrm{N}$, $05.4453^{\circ} \mathrm{E}, 20 \mathrm{~m}, 2018-12-18, \mathrm{~K}$. Rebbas, RK n ${ }^{\circ} 0001$ (ENSA);

Cyclosorus interruptus (Willd.) H. Itô, Algérie, El Tarf, lac Bleu, $36.91069^{\circ} \mathrm{N}, 8.34081^{\circ} \mathrm{E}$, 16 m, G. de Bélair, 1996-10-06, ENSA/GDB n029_14; 1990-10-01, ENSA/GDB n033_06; 1993-09-15, ENSA/GDB n033_08; 1997-11-03, ENSA/GDB n075_19;

Cyclosorus interruptus (Willd.) H. Itô, Algérie, El Tarf, aunaie Oum el Āgareb, $36.82189^{\circ} \mathrm{N}, 8.20965^{\circ} \mathrm{E}, 15 \mathrm{~m}, 1990-10-26$, G. de Bélair, ENSA/GDB n033_07;

Cyclosorus interruptus (Willd.) H. Itô, Algérie, El Tarf, aunaie Bou Glès, $36 . \overline{8} 2679^{\circ} \mathrm{N}$, 8.21664ํ․ $22 \mathrm{~m}$, G. de Bélair, 1992-05-08, ENSA/GDB n033_09; 1991-06-23, ENSA/GDB n033_12; 2005-12-23, GDB ph013_10; 2005-12-23, GDB ph013_11;

Cyclosorus interruptus (Willd.) H. Itô, Algérie, El Tarf, aunaie Righia 2, $36.85339^{\circ} \mathrm{N}$, 8.18883 E, 22 m, 1989-06-19, G. de Bélair, ENSA/GDB n033_10;

Cyclosorus interruptus (Willd.) H. Itô, Algérie, Skikda, aunaie Demnat Ataoua, $36.88987^{\circ} \mathrm{N}, 7.25918^{\circ} \mathrm{E}, 38 \mathrm{~m}, 1990-05-01$, G. de Bélair, ENSA/GDB n033_11;

Thelypteris palustris Schott, Algérie, El Tarf, garaet Esstah, $36.84253^{\circ} \mathrm{N}, 7.98214^{\circ} \mathrm{E}, 5$ m, G. de Bélair, 1996-12-02, ENSA/GDB n029_15; 1993-09-05, ENSA/GDB n033_13; 1991-09-05, ENSA/GDB n033_16; 1992-06-15, ENSA/GDB n033_18; 1992-09-25, ENSA/GDB n033_19; 1995-12-15, ENSA/GDB n033_20; 1996-1202, ENSA/GDB n ${ }^{\circ} 033 \_21$;

Thelypteris palustris Schott, Algérie, Skikda, aunaie Demnat Ataoua, $36.88987^{\circ} \mathrm{N}$, $7.25918^{\circ} \mathrm{E}, 38 \mathrm{~m}$, G. de Bélair, 1991-09-21, ENSA/GDB n033_15; 1993-06-07, ENSA/GDB n033_14; 1991-11-24, ENSA/GDB n033_17; 2014-06-13, ENSA/GDB n ${ }^{\circ} 108 \_25 ; 1993-06-07$, GDB ph013_12; 2011-05-14, GDB ph013_13; 2012-12-21, GDB ph013_14; 2012-12-21, GDB ph013_15; 2014-06-13, GDB ph015_47;

Thelypteris palustris Schott, Algérie, El Tarf, Goureiate, $36.85388^{\circ} \mathrm{N}, 8.1 \overline{7392}{ }^{\circ} \mathrm{E}, 25 \mathrm{~m}$, 1998-08-30, G. de Bélair, ENSA/GDB n075_17; 


\section{Description taxonomique}

Christella dentata a des feuilles en touffes, de 30-70 $\mathrm{cm}$ de longueur, à pétiole assez court; limbe fortement pubescent (sur les faces ainsi que sur les axes) nettement réduit à la base, à texture rêche; pennes incisées jusqu'à la moitié de la longueur des pinnules, cellesci entières et à sommet arrondi. Nervation très particulière, la nervure basale de chaque pinnule s'unissant à celle de la pinnule voisine et formant une nervure verticale qui rejoint le sinus. Un très léger dimorphisme entre les feuilles stériles et fertiles, ces dernières plus longues et dressées, à pennes plus étroites. Sore ronds, à indusie réniforme et pubescent, les pédicelles des sporanges portant des glandes microscopiques oranges. Risque de confusion avec Oreopteris limbosperma et avec les Dryopteris du groupe de la Fougère-mâle; la pilosité du limbe et la nervation de $C$. dentata permettent de trancher. Plante vivace à développement printanier et fructification estivale; les feuilles persistent tout l'hiver et se dessèchent au cours du printemps suivant (Prelli 2001).

Cette espèce est facilement confondue avec C. parasitica (= Cyclosorus parasiticus) (Wagner 1950). Les plantes vivantes de C. parasitica sont plus pâles et d'un vert plus terne, et ont des pennes proximales plus étroites. Plus important encore, ses frondes ne sont pas dimorphes et n'ont pas la rupture nette entre les frondes stériles et fertiles comme $C$. dentata. Les feuilles stériles de $C$. dentata sont plus larges, plus courtes et plus étalées que les autres, et généralement entièrement dépourvues de sores. Une clé des espèces chinoises du sous-genre Cyclosoriopsis (incluant à la fois Cyclosorus parasiticus et Christella dentata) est fournie par Li \& al. (2013).

La séparation des deux genres Christella et Cyclosorus quant à elle, se justifie dans la mesure où l'on suit la récente synthèse du Pteridophyte Phylogeny Group (2016), dans laquelle le genre Christella est maintenu indépendant des Cyclosorus.

\section{Répartition géographique}

Chirstella dentata est originaire des régions tropicales de l'Ancien Monde (répandues en Asie et en Afrique) et des régions tempérées d'Australie et de Nouvelle-Zélande, mais est adventive en Amérique du Nord et en Amérique centrale. Il a été décrit comme ayant une distribution cosmopolite tropicale, mais il se rencontre aussi dans des sites subtropicaux qui ne gèlent pas ou presque, survivant peut-être à quelques gelées par an dans le nord de la Floride (Iwatsuki 1963 ; McCarthy 1998).

C. dentata a été observée pour la première fois au Japon dans les années 1950 et s'est depuis propagée vers le nord (probablement en tant que contaminant de serre) à raison de $3 \mathrm{~km}$ par an, persistant pendant les hivers (Murakami \& al. 2007). À Hawaii, l'espèce a été récoltée beaucoup plus tôt et a probablement été introduite et établie à la fin des années 1800 (MacCaughey 1918; Wagner 1950).

C. dentata est parfois considéré comme indigène dans l'île Lord Howe, de l'île Norfolk, des Tonga et de Tahiti, bien qu'une publication suggère qu'elle a été introduite à Tahiti (Iwatsuki 1963, Rodd \& Pickard 1983 ; Murdock \& Smith 2003 ; Meyer \& al. 2009). Il a également été observé en Nouvelle-Calédonie, au Vanuatu, au Samoa et aux Îles Cook (CABI 2018). 
En Europe, $C$. dentata est présente et indigène dans les régions suivantes : en Grèce sur l'île de Crète, au Portugal aux Azores et à Madère, en Espagne aux Canaries et sur la péninsule (Brownsey \& Jermy, 1973 ; Prelli 2001). Elle est considérée comme douteuse au Maroc (Dobignard \& Chatelain 2010) tandis qu'en Sicile et Italie péninsulaire elle est tantôt considérée comme douteuse (Pignatti 2017) tantôt comme xénophyte occasionnelle (Galasso \& al. 2018), mais sans justification spécifique.

Sa présence en Afrique du Nord dans un site sauvage, en bon état de conservation, riche en espèces subtropicales (Pteris vittata, Pteris cretica, etc.), en plein cœur d'une région historiquement inexplorée sur le plan botanique, nous incite à la considérer comme parfaitement indigène et en position de relique.

\section{Clé d'identification des Thelypteridaceae d'Afrique-du-Nord}

Etant donné qu'aucun pays méditerranéen ni aucune rive continentale de la méditerranée ne présentait jusqu'à ce jour les deux espèces Cyclosorus interruptus et Christella dentata, aucune clé complète ne permet de les distinguer dans les flores usuelles en Europe et en Afrique du Nord. C'est en revanche le cas dans la flore de Chine, très riche en espèces du genre Cyclosorus sensu lato (Youxing \& al. 2013).

Nous fournissons ici une clé des trois espèces de Thelypteridaceae connues en Afrique du nord continentale (Macaronésie exclue), et basée sur le modèle de la flore d'Algérie de Quézel \& Santa (1962 : 24).

1. Feuilles adultes glabres (les jeunes feuilles sont légèrement poilues et glanduleuses). Pinnules minces, à nervures bien visibles par transparence, divisées jusqu'à la nervure. - Marais et bois marécageux - RR: K3: plaine des Guerbès-Senhadja (Ben Azzouz), mares de la Mafragh (Berrihane) - Subcosm. - (= Polystichum thelypteris (L.) Roth ; Nephrodium thelypteris (L.) Desv. ; Dryopteris thelypteris (L.) A. Gray):

Thelypteris palustris Schott

1 '. Feuilles densément poilues-glanduleuses en-dessous. Pinnules coriaces, à nervures peu visibles par transparence, à lobes plus ou moins profond mais n'atteignant jamais la nervure. Indusie couverte de poils denses :

2. Feuilles adultes à limbe démarrant nettement à la base. Rachis des pennes avec grandes écailles isolées à la face inférieure. Pinnules divisées jusqu'à la moitié ou moins, avec 6 à 10 paries de nervures [Fig. 3a] - Marais et bois marécageux - R: K3: lacs et mares de La Calle à la Mafragh (El Kala, Bouteldja, Berrihane), Plaine des Guerbès-Senhadja (Ben Azzouz) - Trop. - « Maas »-(= Nephrodium unitum subsp. callense Trab.; Polypodium unitum Desf., non L. ; Dryopteris gongyloides (Schkuhr) Kuntze subsp. propinqua (R. Br.) Christ.) : .............................. Cyclosorus interruptus (Willd.) H. Itô

2'. Feuilles adultes à limbe progressivement rétréci à la base. Rachis des pennes sans écaille. Pinnules divisées jusqu'à la moitié ou plus, avec 6 à 8 paries de nervures [Fig. 3b] - Bords de ruisseaux ombragés - RR: K2: Oued Ziama, Oued Taza - Subtrop. - (= Thelypteris dentata (Forssk.) E.P. St. John ; Cyclosorus dentatus (Forssk.) Ching) 


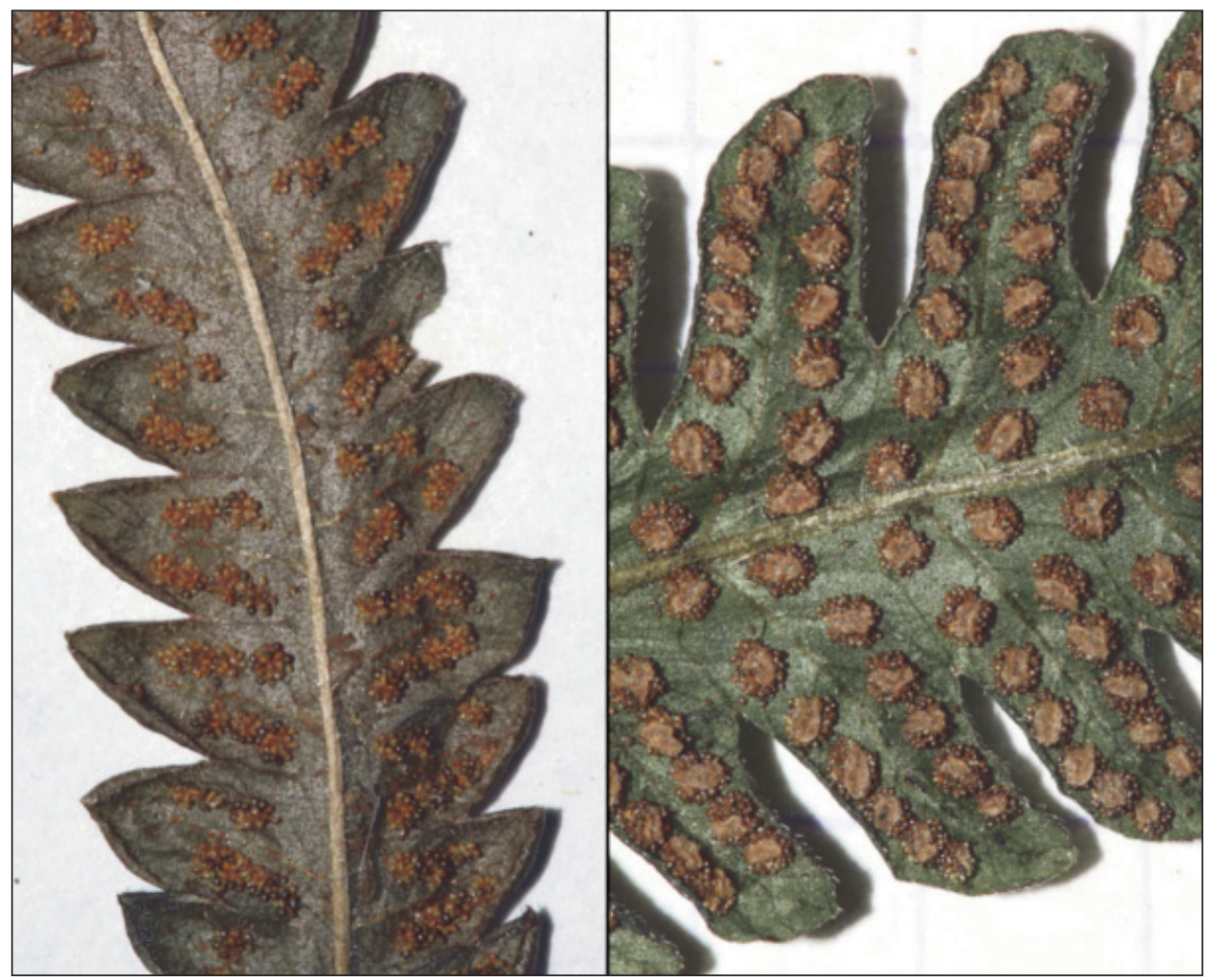

Fig. 3a, 3b. Comparaison entre Cyclosurus interruptus (à gauche) avec écailles brunâtres éparpillées le long du rachis (photo E. Vela, legit G. de Bélair, Lac Bleu, 15.09.1993, GDB n033_08) et Christella dentata (à droite) avec absence d'écaille brunâtre le long du rachis (photo E. Vela, legit K. Rebbas, Oued Ziama, 20.08.2009).

\section{Conclusion}

Tandis que Cyclosorus interruptus est confirmée pour l'Algérie dans la Numidie littorale (K3) où elle côtoie Thelypteris palustris, Christella dentata est une nouveauté pour la flore d'Afrique du Nord. Cette découverte enrichit davantage la ptéridoflore algérienne et vient relancer l'intérêt de la rechercher également au Maroc, notamment dans la péninsule tingitane et le Rif, tandis que Cyclosorus interruptus est signalée dans le Maroc atlantique nord (secteur « Man ») où elle côtoie là aussi Thelypteris palustris (Fennane \& al. 1999).

Ce secteurs méconnus de l'oued Ziama apparaît en continuité entre deux zones importantes pour les plantes (ZIP), celle du parc national de Taza, dont fait partie l'oued Taza, et la ZIP mitoyenne de Babors-Kefrida (Yahi \& al. 2012; Bouchibane \& al. 2017 ; Benhouhou \& al. 2018). La poursuite de ce travail visera l'exploration botanique d'autres secteurs de la Kabylie. Une généralisation des recherches sur les ptéridophytes de toute l'Algérie sera nécessaire à leur clarification taxonomique et nomenclaturale. 


\section{Remerciements}

Nous tenons à remercier vivement les personnes qui nous ont accompagnés sur le terrain, Nacereddine Rebbas, Youcef Rebbas, Saadane Boujerda et Abdelouahab Bouchareb, ainsi que Salima Benhouhou pour le dépôt dans l'herbier ENSA et la consultation de l'herbier GDB.

\section{Références}

Agence nationale des ressources hydrauliques (ANRH) 1993: Carte pluviométrique de l'Algérie du Nord au 1/500 000. Notice explicative. - Alger.

Benhouhou, S., Yahi, N. \& Véla, E. 2018: Algeria. - Pp. 53-60 in: Valderrábano, M., Gil, T., Heywood, V., Montmollin de, B. (eds), Conserving wild plants in the South and East Mediterranean region (chapter 3 "Key Biodiversity Areas (KBAs) for plants in the Mediterranean region "). - Gland.

Bouchibane, M., Vela, E., Bougaham, A. F., Zemouri, M., Mazouz, A. \& Sahnoune, M. 2017: Étude phytogéographique des massifs forestiers de Kéfrida, un secteur méconnu de la zone importante pour les plantes des Babors (Nord-Est algérien). - Rev. Ecol. (la Terre \& la Vie) 72(4): 374-386.

Brownsey, P. J. \& Jermy, A. C. 1973: A Fern Collection Expedition to Crete. - British Fern Gazette 10(6): 331-348.

CABI (Centre for Agriculture and Biosciences International) 2018: Invasive Species Compendium. Christella dentata (Forssk.) Brownsey \& Jermy. https://www.cabi.org/isc/datasheet/113279. [Last Accessed 09.12.2018]

Daoud-Bouattour, A., Muller, S. D., Ferchihi, Ben Jamaa, H., Ghrabi-Gammar, Z., Rhazi, L., Gammar, A. M., Karray, M. R., Soulie-Marsche, I., Zouaidia, H., De Belair, G., Grillas, P. \& Ben Saad-Limam, S. 2009: Recent discovery of the small pillwort (Pilularia minuta Durieu, Marsileaceae) in Tunisia: Hope for an endangered emblematic species of Mediterranean temporary pools? - Comptes Rendus Biol. 332: 886-897. https://doi.org/10.1016/ j.crvi.2009.07.004

Daget, P. 1977: Le bioclimat méditerranéen: analyse des formes climatiques par le système d'Emberger. - Vegetatio 34(2): 87-103.

De Belair, G. \& Vela, E., 2011: Découverte Nymphoides peltata (Gmel) O.Kuntze (Menyanthaceae) en Afrique du Nord (Algérie). - Poiretia 3: 1-7.

Dobignard, A. \& Chatelain, C. 2010: Index synonymique de la flore d'Afrique du Nord, 1. - Genève. — \& - 2013: Index synonymique de la flore d'Afrique du Nord, 5. - Genève.

El Mokni, R., Domina, G., Sebei, H. \& El Aouni, M. H. 2015a: On the distribution and subspecific variation of the Tunisian-Algerian endemic Delphinium sylvaticum (Ranunculaceae) Nordic J. Bot. 33(5): 548-554. http://dx.doi.org/10.1111/njb.00900

- , - - - \& - 2015b: Taxonomic notes and distribution of taxa of Orobanche gr. minor (Orobanchaceae) from Tunisia - Acta Bot. Gallica 162(1): 5-10. http://dx.doi.org/10.1080/12538078.2014.993424

Fennane, M., Ibn Tattou, M., Mathez, J., Ouyahya, A. \& El Oualidi, J. 1999: Flore pratique du Maroc. Manuel de Détermination des Plantes Vasculaires, 1. - Rabat.

Flora of North America Editorial Committee 1993: Flora of North America north of Mexico, 2. New York.

Galasso, G, Conti, F., Peruzzi, L., Ardenghi, N. M. G., Banfi, E., Celesti-Grapow, L., Albano, A., Alessandrini, A., Bacchetta, G., Ballelli, S., Bandini Mazzanti, M., Barberis, G., Bernardo, L., Blasi, C., Bouvet, D., Bovio, M., Cecchi, L., Del Guacchio, E., Domina, G., Fascetti, S., Gallo, L., Gubellini, L., Guiggi, A., Iamonico, D., Iberite, M., Jiménez-Mejías, P., Lattanzi, 
E., Marchetti, D., Martinetto, E., Masin, R.R., Medagli, P., Passalacqua, N. G., Peccenini, S., Pennei, R., Pierini, B., Podda, L., Poldini, L., Prosser, F., Raimondo, F. M., Roma-Marzio, F., Rosati, L., Santangelo, A., Scoppola, A., Scortegagna, S., Selvaggi, A., Selvi, F., Soldano, A., Stinca, A., Wagensommer, R. P., Wilhalm, T. \& Bartolucci, F. 2018: An updated checklist of the vascular flora alien to Italy. - Pl. Biosyst. 152: 556-592. http://doi.org/10.1080/11263504.2018.1441197

Gharzouli, R. \& Djellouli, Y. 2005: Diversité floristique des formations forestières et préforestières des massifs méridionaux de la chaîne des Babors (djebel Takoucht, Adrar ou Mellal, Tababort et Babor) Algérie. - J. Bot. Soc. Bot. France 29: 69-75.

Hadji, K. \& Rebbas, K. 2014: Redécouverte d'Ophrys mirabilis, d'Ophrys funerea et d'Ophrys pallida à Jijel (Algérie). - J. Europ. Orchid. 46(1): 67-78.

Haou, S., De Belair, G. \& Viane, R. 2011: Inventory of the ferns (Filicopsida) of Numidia's (NorthEastern Algeria). - Int. J. Biodiv. Conserv. 3(6): 206-223.

Iwatsukin, K. 1963: New or Noteworthy Ferns from the Tonga Islands. - American Fern J. 53(4): $133-138$.

Jahandiez, E. \& Maire, R. 1931: Catalogue des plantes du Maroc (Spermatophytes et Ptéridophytes), 1. - Alger.

Laribi, M., Acherar, M., Derridji, A. \& Mathez, J. 2009: Nardus stricta L., espèce nouvelle pour la flore algérienne. - J. Bot. Soc. Bot. France 48: 3-6.

—, Acherar, M., Mathez, J. \& Derridji, A. 2011: Découverte Rhynchocorys elephas (L.) Griseb dans l'Akfadou (Grande Kabylie, Algérie): première mention pour Afrique du Nord. - J. Bot. Soc. Bot. France 53: 31-36.

Li, Z. Y., He, Z. R. \& Zhang, X. C. 2013: A taxonomic revision of Cyclosorus subgenus Cyclosoriopsis (Thelypteridaceae) from China. - J. Syst. Evol. 51(5): 609-638. https://doi.org/10.1111/jse.12013

Maire, R. (ed.) 1952: Flore de l'Afrique du Nord, 1. - Paris.

Martin, R. 2008: Des «nouvelles» de Tunisie. - Bull. Soc. Française Orchidophilie Rhône-Alpes 18: $26-31$.

MacCaughey, V. 1918: An Ecological Survey of the Hawaiian Pteridophytes. - J. Ecol. 6(3): 199-219.

McCarthy, P. M. 1998: Flora of Australia, 48. - Melbourne.

Meyer, J. Y., Fourdrigniez, M. \& Taputuarai, R. 2009: Evaluation of terrestrial biodiversity of the island of Me'eti'a (Society archipelago). - Paris.

Murdock, A.G. \& Smith, A. R. 2003: Pteridophytes of Moorea, French Polynesia, with a new species, Tmesipteris gracilis (Psilotaceae). - Pacific Sci. 57(3): 253-265. https://doi.org/10.1353/psc.2003.0024

Murakami, K., Matsui, R., Morimoto, Y. 2007: Northward invasion and range expansion of the invasive fern Thelypteris dentata (Forssk.) St. John into the urban matrix of three prefectures in Kinki District, Japan. - Amer. Fern J. 97(4): 186-198. https://doi.org/10.1640/00028444(2007)97[186:NIAREO]2.0.CO;2

Ouarmim, S., Dubset C. \& Vela, E. 2013: Morphological and ecological evidence for a new infraspecific taxon of the wallflower Erysimum cheiri (Brassicaceae) as an indigenous endemism of the southwestern Mediterranean. - Turk. J. Bot. 37(6): 1061-1069.

Pignatti, S. 2017: Flora d'Italia, $2^{\circ}$ ed., 1. - Milano.

Pteridophyte Phylogeny Group 2016: A community-derived classification for extant lycophytes and ferns. - J. Syst. Evol. 54(6): 563-603. https://doi.org/10.1111/jse.12231

Prelli, R. 2001: Les fougères et plantes alliées de France et d'Europe occidentale. - Belin.

Quézel, P. \& Santa, S. 1962-1963: Nouvelle flore de l'Algérie et des régions désertiques méridionales, 1-2. - Paris. 
Radford, E. A., Catullo, G. \& De Montmollin, B. 2011: Important Plant Areas of the south and east Mediterranean : priority sites for conservation. -Málaga.

Rebbas, K. 2014: Développement durable au sein des aires protégées algériennes, cas du Parc National de Gouraya et des sites d'intérêt biologique et écologique de la région de Bejaia. Thèse doct. - Univ. Sétif, Algérie.

— \& Vela, E. 2008: Découverte d'Ophrys mirabilis P. Geniez \& F. Melki en Kabylie (Algérie). Monde Plantes 496: 13-16.

Rodd, A. N. \& Pickard, J. 1983: Census of Vascular Flora of Lord Howe Island. - Cunninghamia 1(2): $267-280$.

Véla, E. \& Benhouhou, S. 2007: Évaluation d'un nouveau point chaud de biodiversité végétale dans le Bassin méditerranéen (Afrique du Nord). - Compt. Rendus Biol. 330: 589-605. https://doi.org/ 10.1016/j.crvi.2007.04.006

—, Bougaham, A.-F. \& Moulai, R. 2012a: Découverte d'Allium commutatum Guss. (Alliaceae) en Algérie. - Lagascalia 32: 291-295.

—, Telailia, S., Boutabia-Telailia, L. \& De Bélair, G. 2012b: Découverte de Sixalix farinosa (Coss.) Greuter et Burdet (Dipsacaceae) en Algérie. - Lagascalia 32: 284-290.

-, Martin, R. \& Ouni, R. 2015: Ophrys pseudomigoutiana (Orchidaceae), a new species from Tunisia. - Acta Bot. Gallica 162(4): 255-261. https://doi.org/ 10.1080/12538078.2015.1099468

Wagner, W. H. 1950: Ferns Naturalized in Hawaii. - Occasional Papers Bishop Mus. 20(8): 95-120.

Yahi, N., Vela, E., Benhouhou, S., De Belair, G., \& Gharzouli, R. (2012). Identifying important plants areas (key biodiversity areas for plants) in northern Algeria. - J. threatened taxa 4(8): 2753-2765.

Youxing, L., Zhongyang, L. \& Iwatsuki, K. 2013: Cyclosorus Link. - Pp. 372-386 in: Wu, Z. Y., Raven, P. H. \& Hong, D. Y. (eds), Flora of China, 2-3. - Beijing, St. Louis.

Adresses des auteurs:

Khellaf Rebbas ${ }^{1}$, Errol Véla ${ }^{2}$, Abdelazize Franck Bougaham ${ }^{3}$, Abdel Kader Belharat ${ }^{3}$, Gérard De Belair ${ }^{4} \&$ Rémy Prelli ${ }^{5}$,

${ }^{1}$ Département des sciences de la nature et de la vie, faculté des sciences, université Mohamed Boudiaf de M'Sila, Algérie. Laboratoire d'Agro-Biotechnologie et de nutrition en zones arides et semi arides/Equipe de recherche de gestion des ressources naturelles et environnement. Université Ibn Khaldoun, Tiaret, Algérie. Courriel: rebbas.khellaf@gmail.com; khellaf.rebbas@univ-msila.dz

${ }^{2}$ AMAP (botAnique et Modélisation de l'Architecture des Plantes et des végétations), Université de Montpellier / CIRAD / CNRS / INRA / IRD, Montpellier, France. Courriel: errol.vela@cirad.fr

${ }^{3}$ Laboratoire d'Ecologie et Environnement, Université Aberrahmane Mira, Targa Ouzemour-Bejaia, 06000, Algérie. Courriel: abdellazizbougaham@yahoo.fr; belharratabdelkader@gmail.com

${ }^{4}$ Université Badji Mokhtar d'Annaba: BP 533, 23000 Annaba, Algérie. Courriel: grerad_de_belair@yahoo.com

532 rue des Salles-Plestan. 22400 Lamballe, France. Courriel: r-prelli@orange.fr 